\title{
Robotic assistance in interventional radiology: dream or reality?
}

\author{
Vania Tacher ${ }^{1,2,3} \cdot$ Thierry de Baere ${ }^{4,5}$
}

Received: 17 October 2019 / Accepted: 23 October 2019 / Published online: 4 December 2019

(C) European Society of Radiology 2019

\section{Key Points}

- Robotic assistance in medicine has been a revolution.

- The use of robots has naturally extended to interventional radiology for percutaneous and endovascular interventions.

- The guidance is mainly performed with a remote (computer or joystick) allowing the operator and his team not to be exposed to $X$-rays.

Interventional radiology has dramatically increased since its advent in 1953 [1]. Image guidance, initially based on X-rays, became more sophisticated with digital imaging subtraction, MD-CT, CB-CT, and multimodality image fusion, and even PET-CT guidance. Dedicated software for 3D imaging guidance were developed like automatic feeder detection and $3 \mathrm{D}$ needle path trajectory, with the addition of electromagnetic or optic guidance for needle navigation.

Robotic assistance in medicine has been a revolution. It started with a stereotaxic brain tumor biopsy in 1988 based on computed tomography images [2]. It progressively reached multiple surgical applications, like robotic assistance in cardiac surgery for aneurysm and large atrial septum defect repair by mean of endoscopic manipulations in 1988 [3]. The technique was promising as endo instruments offered five degrees of freedom compared with the seven degrees of freedom of the physician's hand. The greatest impact of robotic surgery is increased precision and accuracy during tissue manipulation. Conversely, the cost is an initial lack of depth perception and

Vania Tacher

vania.tacher@aphp.fr

Thierry de Baere

Thierry.DEBAERE@ gustaveroussy.fr

1 Service d'imagerie médicale, CHU Henri Mondor, Créteil, France

2 Université Paris Est, Créteil, France

3 INSERM U955, Equipe 18, Institut Mondor de Recherche Biomédicale, Créteil, France

4 Service de radiologie interventionnelle, Gustave Roussy, Villejuif, France

5 Université Paris-Saclay, Saint-Aubin, France pressure feedback. In 2000, a surgical operation was performed from another continent by an operator who remotely controlled the robot [4]. Since then, the robot Da Vinci (Intuitive Surgical) has been routinely used for various applications in cardiothoracic surgery (coronary bypass), hepatobiliary surgery (hepatectomy, cholecystectomy), general surgery (colectomy), urology (prostatectomy), neurosurgery (tumor resection, external ventricular drainage), and gynecology (hysterectomy). Other robots are dedicated to orthopedic surgery like Rosa (MedTech Surgical) [5].

The use of robots has naturally extended to interventional radiology for percutaneous and endovascular interventions. First-generation of robots helped with the orientation of the needle with two degrees of freedom. Then, robots gained up to six degrees of freedom and extended intervention possibilities. The AcuBot robot developed in the URobotics Laboratory at Johns Hopkins Medical Institutions offered a 2-mm precision. Its use was feasible, safe, and fast for biopsy or percutaneous ablation of various organs [6]. The guidance is mainly performed with a remote (computer or joystick) allowing the operator and his team not to be exposed to X-rays. Hiraki et al report in the current issue the first-in-human experience of Zerobotic (Meicalnet) needle guidance under CT fluoroscopy for biopsy in ten patients [7]. The team reported feasibility and safety. All biopsies performed at various sites were contributive. Mean fluoroscopy time was 29 s (range, 4-124 seconds), robotic intervention, $4 \mathrm{~min}$ (range, 0-23 $\mathrm{min}$ ); and total intervention time, $29 \mathrm{~min}$ (range, 17-53 $\mathrm{min}$ ). This is compliant with the clinical routine, even if we can already foresee that robot installation time is one of the weaknesses.

The Magellan robotic catheter system from Hansen Medical has been evaluated for endovascular aortic repair and various transarterial embolizations [8-10]. Its use may 
be combined with image fusion guidance as pre-intervention 3D-computed tomography angiography overlaid onto live 2D fluoroscopy [11]. Remote catheter control allows to reduce the radiation exposure of the medical team. The force-sensing technology that helps manage the contact force and provides some "palpation effect" has been added to enhance the information provided by the robot to the physician.

In vascular navigation, robotic has still some major drawbacks such as a limited choice of catheter shapes, too large catheters, and their relative stiffness when compared with standard catheters used today. Moreover, the cost of the robot itself and of the catheters, as well as installation time still remain important issues.

Artificial intelligence will potentially help. There is ongoing research in this direction: Fagogenis et al reported an in vivo animal study of autonomous intra-cardiac robotic. The catheter used haptic vision, a hybrid sense using imaging for both touch-based surface identification and force sensing, to follow the wall inside the blood-filled heart [12]. This might avoid the risk of excessive manipulation and tissue damage.

Robotics in interventional radiology is at the beginning of an exciting story, but there is still a long way to go. Within the next few years, robots will probably use straight highways like needle guidance, but more time will be needed before they will be able to help the interventional radiologist drive through tortuous countryside roads as routinely faced in endovascular navigation.

Funding information The authors state that this work has not received any funding.

\section{Compliance with ethical standards}

Guarantor The scientific guarantor of this publication is Thierry de Baere.

Conflict of interest The authors of this manuscript declare no relationships with any companies, whose products or services may be related to the subject matter of the article.

Statistics and biometry No complex statistical methods were necessary for this paper.

Informed consent Approval from the institutional animal care committee was not required because this is an Editorial Comment.
Ethical approval Institutional Review Board approval was not required because this is an Editorial Comment.

\section{References}

1. Seldinger SI (1953) Catheter replacement of the needle in percutaneous arteriography; a new technique. Acta Radiol 39:368-376

2. Kwoh YS, Hou J, Jonckheere EA, Hayati S (1988) A robot with improved absolute positioning accuracy for $\mathrm{CT}$ guided stereotactic brain surgery. IEEE Trans Biomed Eng 35:153-160

3. Carpentier A, Loulmet D, Aupecle B et al (1998) Computer assisted open heart surgery. First case operated on with success. C R Acad Sci III 321:437-442

4. Lee BR, Png DJ, Liew L et al (2000) Laparoscopic telesurgery between the United States and Singapore. Ann Acad Med Singap 29:665-668

5. Sayari AJ, Pardo C, Basques BA, Colman MW (2019) Review of robotic-assisted surgery: what the future looks like through a spine oncology lens. Ann Transl Med 7:224

6. Solomon SB, Patriciu A, Bohlman ME, Kavoussi LR, Stoianovici D (2002) Robotically driven interventions: a method of using CT fluoroscopy without radiation exposure to the physician. Radiology. 225:277-282

7. Hiraki T, Kamegawa T, Matsuno T, Komaki T, Sakurai J, Kanazawa S (2018) Zerobot $®$ : a remote-controlled robot for needle insertion in CT-guided interventional radiology developed at Okayama University. Acta Med Okayama 72:539-546

8. Riga CV, Bicknell CD, Rolls A, Cheshire NJ, Hamady MS (2013) Robot-assisted fenestrated endovascular aneurysm repair (FEVAR) using the Magellan system. J Vasc Interv Radiol 24:191-196

9. Del Giudice C, Pellerin O, Nouri Neville M et al (2018) Comparison of two endovascular steerable robotic catheters for percutaneous robot-assisted fibroid embolization. Cardiovasc Intervent Radiol 41:483-488

10. Rolls AE, Riga CV, Bicknell CD, Regan L, Cheshire NJ, Hamady MS (2014) Robot-assisted uterine artery embolization: a first-inwoman safety evaluation of the Magellan System. J Vasc Interv Radiol 25:1841-1848

11. Cochennec F, Kobeiter H, Gohel M et al (2015) Feasibility and safety of renal and visceral target vessel cannulation using robotically steerable catheters during complex endovascular aortic procedures. J Endovasc Ther 22:187-193

12. Fagogenis G, Mencattelli M, Machaidze Z et al (2019) Autonomous robotic intracardiac catheter navigation using haptic vision. Sci Robot 4:eaaw1977

Publisher's note Springer Nature remains neutral with regard to jurisdictional claims in published maps and institutional affiliations. 